McGill Law Journal

Revue de droit de McGill

\title{
Emmanuelle Jouannet, Hélène Ruiz Fabri et Jean-Marc Sorel, dir., Regards d'une génération de juristes sur le droit international, Paris, Pedone, 2008, pp. 462
}

\section{Olivier Barsalou}

Volume 55, numéro 4, november 2010

URI : https://id.erudit.org/iderudit/1000790ar

DOI : https://doi.org/10.7202/1000790ar

Aller au sommaire du numéro

Éditeur(s)

McGill Law Journal / Revue de droit de McGill

ISSN

0024-9041 (imprimé)

1920-6356 (numérique)

Découvrir la revue

Citer ce compte rendu

Barsalou, O. (2010). Compte rendu de [Emmanuelle Jouannet, Hélène Ruiz Fabri et Jean-Marc Sorel, dir., Regards d'une génération de juristes sur le droit international, Paris, Pedone, 2008, pp. 462]. McGill Law Journal / Revue de droit de McGill, 55(4), 967-972. https://doi.org/10.7202/1000790ar d'utilisation que vous pouvez consulter en ligne. 


\begin{abstract}
RECENSION COMPARATIVE-
Emmanuelle Jouannet, Hélène Ruiz Fabri et Jean-Marc Sorel, dir., Regards d'une génération de juristes sur le droit international, Paris, Pedone, 2008, pp. 462.
\end{abstract}

\title{
Olivier Barsalou*
}

\section{Introduction}

Cette ouvrage présente une génération d'internationalistes nés entre 1955 et 1965 à qui on a posé la question suivante: "Quelle est votre vision du droit international à l'aube du XXIème siècle ? "1. Près d'une trentaine de spécialistes du droit international ont répondu à l'appel lancé par les professeurs Emmanuelle Jouannet, Hélène Ruiz Fabri et Jean-Marc Sorel. Produit de la mondialisation, de la révolution des technologies de la communication et de la fin de la guerre froide, cette génération d'internationalistes se découvre dans cet ouvrage au travers de courts articles schématiques ou de brefs récits personnels et anecdotiques.

Notre commentaire s'articule autour de deux thèmes qui se retrouvent dans l'ensemble des vingt-huit contributions à l'ouvrage. Le premier est la perte de certitudes dans la discipline du droit international qui se traduit dans le débat contemporain sur l'unité et la fragmentation du droit international (I). Le second thème porte sur le rôle de la realpolitik et des relations de pouvoir dans le droit international contemporain (II).

\section{La perte des certitudes dans la discipline du droit international : unité et fragmentation}

Enfants de Mai 68, plusieurs des collaborateurs de cet ouvrage ont grandi à une époque où les certitudes et les repères du monde moderne et occidental furent contestés pour être finalement rejetés en bloc par une génération entière. Le droit international n'y a pas échappé. Faisant écho à la réflexion de l'intellectuel français Guy Debord ${ }^{2}$, le professeur Andrea Gattini souligne que le droit international est soumis aux impératifs de la société du spectacle. Mélancolique, le professeur Gattini déplore cette

* Candidat au doctorat en droit, New York University School of Law.

(C) Olivier Barsalou 2010

Citation: (2010) 55 McGill L.J. 967 Référence : (2010) 55 R.D. McGill 967

1 Emmanuelle Jouannet, Hélène Ruiz Fabri et Jean-Marc Sorel, dir., Regards d'une génération de juristes sur le droit international, Paris, Pedone, 2008 aux pp. 3-5.

2 Guy Debord, La société du spectacle, Paris, Gallimard, 1992. 
perte de sens dans la discipline du droit international et la disparition des certitudes.

Cet environnement intellectuel a favorisé le développement de nouvelles approches critiques en droit international fondées sur un profond scepticisme épistémologique face à la discipline classique du droit international. Les écrits des professeurs David Kennedy et Martti Koskenniemi ${ }^{3}$, qui ont exercé une certaine influence sur la pensée de plusieurs contributeurs, dont Andrea Bianchi, Hilary Charlesworth, Emmanuelle Jouannet et Philip Sands, incarnent cette rupture intellectuelle avec les fondements classiques du droit international. À ce sujet, la professeure Jouannet fait écho, dans sa contribution, aux problèmes d'indétermination et d'interprétation des règles dans le droit international général. Ces développements théoriques et méthodologiques ont paradoxalement participé au renouvellement de la discipline du droit international et à l'érosion de ses fondements classiques, soit à la perte de sens qui est identifiée, entre autres, par le professeur Gattini.

Selon le professeur Aeyal Gross, la postmodernité, ou plutôt la perte de sens, ne serait qu'un seul des deux défis auxquels doit faire face le droit international du vingt-et-unième siècle. L'autre serait l'antimodernité, la négation et la corruption de son caractère normatif. Le premier défi identifié par le professeur Gross se traduit actuellement dans le discours sur la fragmentation normative, institutionnelle, disciplinaire et idéologique du droit international. La fragmentation serait le produit de cette perte de sens ou plutôt, pour employer les termes du professeur Alberto do Amaral Jùnior, de la perte de la cohérence systémique dans le droit international. De son côté, le professeur Bianchi semble croire que la fragmentation du droit international est un phénomène sociologique. Selon lui, il y aurait une intensification du processus de spécialisation en droit international allant de pair avec la disparition d'un langage commun et d'une épistémologie unificatrice pour le droit international, image jadis incarnée par le généraliste. La professeure Laurence Boisson de Chazournes semble partager cet avis en soulignant qu'au travers le débat sur la fragmentation et l'unité du droit international, la responsabilité du juriste envers sa discipline et les idéaux qu'elle incarne est souvent omise.

En réponse à cette perte de sens et à la dissolution des certitudes, la professeure Anne Peters voit dans l'indétermination et la fragmentation

3 Voir notamment David Kennedy, The Dark Sides of Virtue: Reassessing International Humanitarianism, Princeton, Princeton University Press, 2004 ; David Kennedy, Of War and Law, Princeton, Princeton University Press, 2006 ; Martti Koskenniemi, From Apology to Utopia: The Structure of International Legal Argument, Helsinki, Finnish Lawyers' Publishing Company, 1989; Martti Koskenniemi, "The Fate of Public International Law: Between Technique and Politics » (2007) 70 Mod. L. Rev. 30 ; Martti Koskenniemi, La politique du droit international, Paris, Pedone, 2007 [Koskenniemi, La politique]. 
du droit la lente édification d'un projet de constitutionnalisation des relations internationales ${ }^{4}$. La contribution de la professeure Peters met en exergue un réflexe partagé par certains des collaborateurs de l'ouvrage. En effet, plusieurs omettent de questionner la légitimité et la validité du projet de constitutionnalisation du droit international : au fond, à quoi sert-il ? La proposition de la professeure Peters s'inscrit dans cette quête d'unité quelque peu illusoire du droit international et obscurcit le rôle du politique dans le discours du droit international ${ }^{5}$. Le professeur Olivier Corten abonde aussi dans ce sens. Selon lui, la question de la constitutionnalisation du droit international demeure une proposition utopique dans la mesure où le droit international ne constitue qu'un " champ " des relations internationales ${ }^{6}$, selon l'expression consacrée par le sociologue français Pierre Bourdieu?

L'illusion du constitutionnalisme, la fragmentation de la discipline du droit international et l'impossible quête de sens et de certitudes attisent le sentiment de crise permanente dans la discipline du droit international ${ }^{8}$. Le catastrophisme constituerait le nouvel ethos de l'internationaliste (Jean-Marc Sorel). Ce dernier serait affligé d'une mélancolie permanente (Mariano J. Aznar Gómez) face au danger causé par le risque d'éclatement qui guetterait le droit international (Jorge Cardona Llorens). Ce pessimisme doit cependant être contextualisé. Par exemple, selon le professeur Nathaniel Berman, le renouvellement de la pensée internationaliste est toujours un symptôme ou un produit des crises qui traversent la société internationale $(1648,1919,1945,1989)$. Le droit international se construirait donc, selon ce dernier, sur les ruines des échecs d'institutionnalisation des rapports internationaux.

Ce pessimisme ambiant n'est toutefois pas partagé par l'ensemble des collaborateurs de l'ouvrage. Pour le professeur Giuseppe Cataldi, le droit international est dans une période de transition. La professeure Jouannet se rallie d'ailleurs à cette idée. Selon elle, le droit international entre dans une nouvelle phase de développement qui s'inscrit dans la continuité et non dans la rupture avec le passé. Cependant, tout comme la majorité de

4 Pour une perspective historique sur l'alternance entre le discours sur la fragmentation et celui sur la constitutionnalisation du droit international, voir Anne-Charlotte Martineau, "The Rhetoric of Fragmentation: Fear and Faith in International Law" (2009) 22 Leiden J. Int'l L. 1.

5 Koskenniemi, La politique, supra note 3.

6 Pour une application de la pensée de Pierre Bourdieu aux relations internationales, voir Frédéric Mérand et Vincent Pouliot, « Le monde de Pierre Bourdieu : éléments pour une théorie sociale des Relations internationales » (2008) 41 Rev. can. sci. pol. 603.

7 Pierre Bourdieu avec Loïc J.D. Wacquant, Réponses : pour une anthropologie réflexive, Paris, Seuil, 1992 aux pp. 71-91.

8 Voir Hilary Charlesworth, «International Law: A Discipline of Crisis» (2002) 65 Mod. L. Rev. 377. 
ses confrères et consœurs ayant collaboré à cet ouvrage, elle demeure évasive quant à sa vision du droit international du vingt-et-unième siècle ou l'orientation que doit emprunter la quête de sens de la discipline du droit international. Le futur est noyé dans le flou esthétique et évanescent de la rhétorique internationaliste. Par exemple, le professeur Georg Nolte note sagement que la quête du sens et de l'unité du droit international doit emprunter la voie d'un " informed pragmatism between morality and form $"{ }^{9}$. Il s'agit d'une position plutôt singulière et paradoxale dans les circonstances puisqu'elle se contente d'indiquer vaguement la marche à suivre et non la direction à emprunter.

La proposition du professeur Nolte semble être symptomatique d'une propension, parmi les collaborateurs de cet ouvrage, à s'enfermer dans un formalisme juridique parfois stérile, inhibiteur et paralysant. Le projet de (re)définition de la nature et de l'objet de cette quête du sens profond de la discipline du droit international est donc laissé en pâture aux rationalistes, réalistes et autres négationnistes du droit international ${ }^{10}$.

\section{Le rôle de la realpolitik et des relations de pouvoir}

Cette conclusion préliminaire nous mène au deuxième défi identifié par le professeur Gross, celui de la formulation d'une réponse aux forces de l'antimodernité dans le droit international, celles lui niant toute normativité. Vidé de son sens, le droit international serait donc soumis aux aléas de certains pouvoirs aux prétentions hégémoniques. C'est le constat que font les professeurs Slim Laghmani et Mohamed Salah. Ces deux professeurs partagent une vision commune et pessimiste du statut actuel du droit international. Depuis la fin de la guerre froide, la discipline du droit international a vu l'établissement d'un nouvel ordre politique mondial et l'avènement d'un droit impérial américain ${ }^{11}$. On assisterait, selon les professeurs Laghmani et Salah, à la colonisation du droit international par le droit constitutionnel américain. Cela aurait pour résultat de favoriser l'harmonisation et l'unification du droit international à travers sa cooptation par les États-Unis. Selon le professeur Sorel, l'assujettissement du droit international, voire son assimilation par les puissances hégémoniques occidentales, aurait aussi pour corollaire

9 Georg Nolte, «Between Informed Pragmatism, Morality and Form» dans Jouannet, Ruiz Fabri et Sorel, supra note 1, 277 à la p. 277.

10 Voir Eric A. Posner, The Perils of Global Legalism, Chicago, University of Chicago Press, 2009. La contribution du professeur Sands se penche directement sur ces questions et réfute les arguments avancés par certains juristes américains dont ceux (implicitement) du professeur Posner.

11 Sur cette dernière idée, voir Michael Hardt et Antonio Negri, Empire, Cambridge, (Mass.), Harvard University Press, 2000 aux pp. 160-82. 
l'avènement d'un mode de pensée unique, le libéralisme, niant l'expression des pluralismes ${ }^{12}$.

Dans le même ordre d'idées, la cooptation du droit international contemporain par les puissances occidentales participe, selon le professeur Tony Anghie, à la reproduction et au renouvellement des structures de dépendance et de domination des pays du Tiers-Monde dans la société internationale. Le professeur Anghie raconte l'histoire des illusions perdues des pays du Tiers-Monde face au pouvoir émancipatoire du droit international pour des millions d'individus qui font quotidiennement l'expérience de l'inégalité et de la pauvreté.

Les contributeurs à l'ouvrage étudié ne partagent cependant pas tous le pessimisme affiché par le professeur Anghie. Par exemple, la professeure Charlesworth reconnaît que le droit international est un levier au potentiel émancipatoire latent pour des millions d'individus. Circonspecte, la professeure Charlesworth prend soin de spécifier que le droit international n'est pas intrinsèquement plus efficace ou légitime que les autres formes de régulation. Elle note cependant que le droit a un potentiel transformateur qui demeure encore largement inexploité et inexploré. De son côté, le professeur Gross voit dans le droit international une forme de pouvoir et de contre-pouvoir. Selon certains, la paralysie qui afflige le droit international contemporain est un symptôme de la tension entre domination et émancipation se trouvant au cœur du droit international ${ }^{13}$.

\section{Conclusion}

Le lecteur referme cet ouvrage avec un sentiment d'incomplétude et de vide ; une image floue et abstraite d'un " bilan générationnel mondialisé " du droit international qui ne se matérialise pas, mais que l'on espère toujours. À ce sujet, on déplore l'absence d'une conclusion générale ou d'un essai final d'anthropologie ou de sociologie de la discipline du droit international. Une telle synthèse aurait très certainement contribué à mettre en lumière les similitudes et les différences ainsi qu'à texturer cette image inachevée que nous renvoie l'ouvrage. De plus, celui-ci aurait gagné en force, en subtilité et en cohésion si les différents collaborateurs avaient été en mesure d'adopter une démarche résolument réflexive dans la préparation de leur contribution, puisque la question qui leur a été posée se prêtait justement à ce type d'exercice. La fragmentation et le syncrétisme dus au mélange des différentes visions des collaborateurs auraient constitué les principales forces de cet ouvrage s'ils avaient été

12 Voir aussi Gerry Simpson, "Two Liberalisms» (2001) 12 E.J.I.L. 537. Le professeur Simpson qualifie ce type de libéralisme de « liberal anti-pluralism» (ibid. à la p. 537).

13 Emmanuelle Jouannet, « Universalism and Imperialism: The True-False Paradox of International Law?» (2007) 18 E.J.I.L. 379. 
conjugués avec une question au potentiel unificateur et une ligne éditoriale claire et précise. Ce potentiel a été perdu en route comme en fait foi le caractère plutôt inégal des différentes contributions. On salue toutefois l'éclectisme, la candeur et l'honnêteté de plusieurs contributions dont celle du professeur Djedjro Francisco Meledje qui souligne sans détour les problèmes structuraux ainsi que l'absence de ressources financières et matérielles qui minent le développement de la discipline du droit international en Afrique subsaharienne. Ce type de contributions côtoie toutefois maladroitement certaines autres dont le formalisme et le ton impersonnel cadre difficilement avec la nature et les objectifs poursuivis par cet ouvrage. En terminant, cet ouvrage nous rappelle que le droit international incarne des valeurs et des aspirations diverses, à la fois contradictoires et complémentaires. Surtout, il symbolise cette multitude, ces espoirs en un projet émancipatoire global. 